\title{
Energy cycle and bound of Qi chaotic system
}

\author{
Guoyuan Qi ${ }^{\mathrm{a} *}$, Jiangfeng Zhang ${ }^{\mathrm{b}}$ \\ a Tianjin Key Laboratory of Advanced Technology of Electrical Engineering and Energy, Tianjin \\ Polytechnic University, Tianjin 300387, China \\ ${ }^{\mathrm{b}}$ School of Electrical Mechanical and Mechatronic Systems, University of Technology Sydney, \\ Australia
}

\begin{abstract}
The Qi chaotic system is transformed into a Kolmogorov-type system, thereby facilitating the analysis of energy exchange in its different forms. Regarding four forms of energy, the vector field of this chaotic system is decomposed into four forms of torque: inertial, internal, dissipative, and external. The rate of change of the Casimir function is equal to the exchange power between the dissipative energy and the supplied energy. The exchange power governs the orbital behavior and the cycling of energy. With the rate of change of Casimir function, a general bound and least upper bound of the Qi chaotic attractor are proposed. A detailed analysis with illustrations is conducted to uncover insights, in particular, cycling among the different types of energy for this chaotic attractor and key factors producing the different types of dynamic modes.
\end{abstract}

\section{Introduction}

In the past 20 years, some numerical chaotic models such as the Chen system [1], Lü system [2], Qi chaotic system [3], and some hyperchaotic systems [4, 5] were generated. These systems were constructed via mathematics and simulations whereas the Lorenz system [6] was modeled on a physical process. A few studies have investigated the application of the Lorenz system in meteorology [7] and mechanics [8]. The main research focus of these numerical systems as well as the Lorenz system has been on their dynamic analysis. Topics usually include bound analysis [9], aperiodic solutions, sensitivity to initial conditions, bifurcation theory [10], circuit implementations, 
calculation of Lyapunov exponents [11, 12], fractional order [13], chaos-based communication, proof of chaos existence, chaos control [14], and synchronization. However, these aspects of the research cannot explain the mechanism or reason for the production of dynamic modes, and also cannot interpret the physical analogues of state variables. To explore them, the mechanics of these numerical systems must be investigated. The lines of study include force analysis, physical analogue interpretation, energy transformation between internal energy, and supplied energy. Arnold [15] presented a Kolmogorov system describing a dissipative-forced dynamic system or hydro-dynamic instability with a Hamiltonian function. Pasini and Pelino [16] gave a unified view of the Kolmogorov and Lorenz systems, thereby providing a force analysis of the Lorenz system. The recurrence of dynamics [17] and energy cycling [8] for the Lorenz system were also investigated with the understanding obtained from the Kolmogorov system.

Although the derivation of the Lorenz system is different from these numerical chaotic systems, they all have similar vector fields and chaotic dynamics. Therefore, from the mechanic's point of view, both types of systems must be governed by similar forces. The transformation of a numerical chaotic system into a Kolmogorov system can build a bridge between physical chaotic systems and numerical chaotic systems. Qi and Liang [18] transformed the Qi four-wing chaotic system to a Kolmogorov system, performed a force analysis and interpreted the state of chaos as angular momentum.

Therefore, the Hamiltonian function and the Kolmogorov system provide a starting point in studying the mechanism for these numerical chaotic systems. Furthermore, the Casimir function, like enstrophy or potential vorticity in a fluid dynamic context, is very useful in analyzing stability conditions and global description of a dynamical system. It represents a constant of the motion of the Hamiltonian system; moreover it defines a foliation of the phase space [8, 19]. The energetics of the Lorenz system using the Casimir function has already been studied [20].

The study of energy cycles is important in mechanics, for there is instantaneous exchange among kinetic energy, potential energy, dissipative and supplied energy in physical processes. Each type of energy is represented by a type of force. When multiple energy exchanges operate frequently and substantially, the mechanism becomes complicated, and chaos may arise. Because each type of torque has a corresponding energy, an analysis of energy can reflect the torque characteristics. So far, there has been no study regarding the energy cycle of numerical chaotic systems. 
In this paper, the vector field of the Qi chaotic system is decomposed into inertial torque, internal torque, dissipative torque, and external torque. Correspondingly, kinetic energy, potential energy, dissipative energy, and external energy are identified in the system. The rate of change of the Casimir energy for a Qi chaotic attractor governs the dynamics of the system. The mechanism for different dynamic modes is revealed from the combination of different torques, which explains the physical phenomena and the energy cycles. The bound of the chaotic attractor is given through the extremal points of the Casimir function. Normally, it is difficult to find the bound of a chaotic attractor; even if there is an available method [9], the positive definite matrix is exceedingly difficult to solve in the equation for Lyapunov stability.

The rest of the paper is organized as follows: Section 2 describes the transformation between the Qi chaotic system and the Kolmogorov-type system. Section 3 presents the decomposition of the system's energy into its four forms and analyzes the cycling of energy using the Hamiltonian and Casimir functions; the bound of the chaotic attractor is also proposed. Section 4 analyzes the energy cycling among the different dynamic modes and uncovers the reason for chaos generation. Finally, a conclusion is made.

\section{Transformation of the Qi chaotic system into Kolmogorov system}

The Qi chaotic system is presented in the form [3]

$\dot{x}_{1}=a\left(x_{2}-x_{1}\right)+x_{2} x_{3}$,

$\dot{x}_{2}=c x_{1}-x_{2}-x_{1} x_{3}$,

$\dot{x}_{3}=x_{1} x_{2}-b x_{3}$.

Here, $a, b, c \in R^{+}$are constant parameters of the system.

To discover the physical analogue of the state variables and mechanics of the system, we introduce the Kolmogorov system and the Euler equation. Arnold [15] presented a Kolmogorov system describing dissipative-forced dynamical systems or hydro-dynamic instability, written in 3D form

$\dot{\mathbf{x}}=\{\mathbf{x}, H\}-\Lambda \mathbf{x}+\mathbf{f}$,

where $\mathbf{x}=\left[\begin{array}{lll}x_{1} & x_{2} & x_{3}\end{array}\right]^{\mathrm{T}},\{$,$\} represents the algebraic structure of the kinetic energy part of the$ Hamiltonian function of a system, denoted by $H$, and the Lie-Poisson structure is defined as [21] 
$\{F, G\}=-\mathbf{x} \cdot(\nabla F \times \nabla G)$,

where $F, G \in C^{\infty}\left(\mathbf{g}^{*}\right), \mathbf{g}$ is Lie algebra. The positive definite diagonal matrix $\Lambda$ represents the dissipative force and the last term $\mathbf{f}$ represents the external force.

The Euler equation without external force for an incompressible fluid or a free rigid body gives a Hamiltonian description, which can be written as [21]

$\dot{x}_{1}=\left(\Pi_{3}-\Pi_{2}\right) x_{2} x_{3}$,
$\dot{x}_{2}=\left(\Pi_{1}-\Pi_{3}\right) x_{1} x_{3}$,
$\dot{x}_{3}=\left(\Pi_{2}-\Pi_{1}\right) x_{1} x_{2}$,

where $\Pi_{i}=I_{i}^{-1}, I_{i}$ is the principle moment of inertia for the group $\mathrm{SO}(3)$, and $x_{i}$ is the angular momentum satisfying

$x_{i}=I_{i} \omega_{i}$,

with $\omega_{i}$ the angular velocity. Eq. (4) can be written in the succinct form

$\dot{\mathbf{x}}=\mathbf{x} \times \Pi \mathbf{x}=\mathbf{x} \times \mathbf{\Omega}$,

where $\quad \mathbf{x}=\left[\begin{array}{lll}x_{1} & x_{2} & x_{3}\end{array}\right]^{\mathrm{T}}, \quad \mathbf{\Omega}=\left[\begin{array}{lll}\omega_{1} & \omega_{2} & \omega_{3}\end{array}\right]^{\mathrm{T}}, \quad \boldsymbol{\Pi}=\operatorname{diag}\left(\begin{array}{lll}\Pi_{1} & \Pi_{2} & \Pi_{3}\end{array}\right)$. Decomposing the Hamiltonian

$H=K+U$,

with $U=0$ and

$K=\frac{1}{2}\left(\Pi_{1} x_{1}^{2}+\Pi_{2} x_{2}^{2}+\Pi_{3} x_{3}^{2}\right)$,

and replacing $F$ by $\mathbf{x}$ and $G$ by $H$ in Eq. (3), Eq. (4) is equivalent to [21]

$\dot{F}=\{F, H\}$,

i.e.,

$\dot{\mathbf{x}}=\{\mathbf{x}, H\}=\mathbf{x} \times \boldsymbol{\Pi} \mathbf{x}$.

We find that under a pure inertial force, the Kolmogorov system (2) is the same as the Euler equation (10).

Remark 1

(1) The force (or torque) $\{\mathbf{x}, H\}$ in the Euler equation for a free rigid body is in the form of a 
fictitious force, either the inertial force or the centrifugal force, which consists of quadratic terms.

(2) The Kolmogorov system is a generalized Euler equation with dissipative and external forces.

(3) The quadratic terms are skew-symmetric, i.e., the sum of the coefficients of all quadratic terms (inertial force) in the Lie-Poisson bracket, [Eq. (3) or (10)], is zero.

(4) The Hamiltonian function $H$ in the bracket of the Kolmogorov system only contains the kinetic energy term $K$, i.e., the potential energy vanishes $(U=0)$.

We now establish an analogy between the Qi chaotic system and the Kolmogorov system. Note that the sum of coefficients of all quadratic terms is nonzero in the Qi chaotic system. To satisfy the condition, we introduce the following transformation

$y_{1}=\alpha x_{1}, y_{2}=x_{2}, y_{3}=\beta x_{3}$,

with inverse

$x_{1}=\frac{1}{\alpha} y_{1}, x_{2}=y_{2}, x_{3}=\frac{1}{\beta} y_{3}$,

where $\alpha$ and $\beta$ are nonzero constants. Hence, Eq. (1) is transformed into

$$
\begin{aligned}
& \dot{y}_{1}=\frac{\alpha}{\beta} y_{2} y_{3}-a y_{1}+\alpha a y_{2}, \\
& \dot{y}_{2}=-\frac{1}{\alpha \beta} y_{1} y_{3}+\frac{c}{\alpha} y_{1}-y_{2}, \\
& \dot{y}_{3}=\frac{\beta}{\alpha} y_{1} y_{2}-b y_{3} .
\end{aligned}
$$

We choose parameters $\alpha$ and $\beta$ such that

$$
\frac{\alpha}{\beta}-\frac{1}{\alpha \beta}+\frac{\beta}{\alpha}=\frac{1}{\alpha \beta}\left(\alpha^{2}+\beta^{2}-1\right)=0 .
$$

to satisfy the skew-symmetric requirement of the Lie-Poisson bracket. To determine the potential energy from the Hamiltonian function, we make a further transformation

$$
z_{1}=y_{1}, z_{2}=y_{2}, z_{3}=y_{3}-\gamma,
$$

with inverse

$$
y_{1}=z_{1}, y_{2}=z_{2}, y_{3}=z_{3}+\gamma \text {. }
$$

Eq. (13) is transformed into 
$z_{1}=\frac{\alpha}{\beta} z_{2} z_{3}-a z_{1}+\left(\alpha a+\frac{\alpha \gamma}{\beta}\right) z_{2}$

$\dot{z}_{2}=-\frac{1}{\alpha \beta} z_{1} z_{3}+\left(\frac{c}{\alpha}-\frac{\gamma}{\alpha \beta}\right) z_{1}-z_{2}$,

$\dot{z}_{3}=\frac{\beta}{\alpha} z_{1} z_{2}-b z_{3}-b \gamma$

To meet the skew-symmetric requirement for the potential energy part in the Lie-Poisson bracket, let $\alpha a+\frac{\alpha \gamma}{\beta}=-\left(\frac{c}{\alpha}-\frac{\gamma}{\alpha \beta}\right)$. Then, from Eq. (14), we derive

$\gamma=\frac{c+\alpha^{2} a}{\beta}$

Setting

$e_{1}=\frac{\alpha}{\beta}, e_{2}=-\frac{1}{\alpha \beta}, e_{3}=\frac{\beta}{\alpha}, c_{1}=\alpha a+\frac{\alpha \gamma}{\beta}$,

Eq. (17) can be written as

$\dot{x}_{1}=e_{1} x_{2} x_{3}-a x_{1}+c_{1} x_{2}$,

$\dot{x}_{2}=e_{2} x_{1} x_{3}-c_{1} x_{1}-x_{2}$,

$\dot{x}_{3}=e_{3} x_{1} x_{2}-b x_{3}-b \gamma$,

with

$e_{1}+e_{2}+e_{3}=0$

where the relabeling $x_{1}=z_{1}, x_{2}=z_{2}, x_{3}=z_{3}$ is made for notational convenience.

Denoting the inverse of the principle moments of inertia for Eq. (20) as

$\boldsymbol{\Pi}=\operatorname{diag}\left(\begin{array}{lll}\Pi_{1} & \Pi_{2} & \Pi_{3}\end{array}\right)=\operatorname{diag}\left(1 \quad\left(1+e_{3}\right) \quad\left(1+e_{1}+e_{3}\right)\right)$,

the kinetic part is expressed as

$K=\frac{1}{2}\left(x_{1}^{2}+\left(1+e_{3}\right) x_{2}^{2}+\left(1+e_{1}+e_{3}\right) x_{3}^{2}\right)$.

Setting the potential part as

$U=c_{1} x_{3}$

the Hamiltonian function is

$H=K+U$. 
Note that the Hamiltonian function contains the potential energy for the transformed Qi chaotic system (20). We then evaluate the Lie-Poisson bracket as

$$
\{\mathbf{x}, H\}=\mathbf{x} \times \nabla H=\mathbf{x} \times(\nabla K+\nabla U)=\left[\begin{array}{cc}
e_{1} x_{2} x_{3} & +c_{1} x_{2} \\
e_{2} x_{1} x_{3} & -c_{1} x_{1} \\
e_{3} x_{1} x_{2} & 0
\end{array}\right] .
$$

Eq. (20) can be written as a Kolmogorov system,

$$
\begin{aligned}
\dot{\mathbf{x}} & =\{\mathbf{x}, H\}-\Lambda \mathbf{x}+\mathbf{f} \\
& =\left[\begin{array}{cc}
e_{1} x_{2} x_{3} & +c_{1} x_{2} \\
e_{2} x_{1} x_{3} & -c_{1} x_{1} \\
e_{3} x_{1} x_{2} & 0
\end{array}\right]-\left[\begin{array}{c}
a x_{1} \\
x_{2} \\
b x_{3}
\end{array}\right]+\left[\begin{array}{c}
0 \\
0 \\
-b \gamma
\end{array}\right],
\end{aligned}
$$

where

$$
\Lambda=\left[\begin{array}{ccc}
\Lambda_{1}=a & 0 & 0 \\
0 & \Lambda_{2}=1 & 0 \\
0 & 0 & \Lambda_{3}=b
\end{array}\right], \mathbf{f}=\left[\begin{array}{c}
0 \\
0 \\
-b \gamma
\end{array}\right]
$$

\section{Remark 2:}

(1) The transformed Qi chaotic system (27) is a dynamic system of Kolmogorov type.

(2) The variable $\mathbf{x}$ is analogous to angular momentum, therefore the time derivative $\dot{\mathbf{x}}$ represents the torque of a rigid body or fluid flow.

(3) The Lie-Poisson bracket, $\{\mathbf{x}, H\}$, characterizes the conservative part of the chaotic system. It consists of the inertial torque (quadratic terms) generated by the kinetic energy and internal torque released by the potential.

(4) Terms $c_{1} x_{2}$ and $-c_{1} x_{1}$ are generated from the potential energy $U=c_{1} x_{3}$. They interact in a conservative system. If term $c_{1} x_{2}$ is called torque, then term $-c_{1} x_{1}$ is the reaction torque, which resembles the gravitational force and the reaction force produced by the potential energy between Earth and an object revolving around it. Therefore, the vector $\left[\begin{array}{lll}c_{1} x_{2} & -c_{1} x_{1} & 0\end{array}\right]^{\mathrm{T}}$ is called the internal torque produced by the potential energy, which makes the system more complicated than the Kolmogorov system for which the potential energy is missing [16].

(5) The term $-\Lambda \mathbf{x}$ represents the dissipative torque (force), which could be friction or heat exchange or viscous force. 
(6) The third term is the external torque.

We now consider the system's equilibria. After the two-fold transformation, the number and properties of the equilibria of system (27) remain the same as system (1), but the locations of these equilibria are transformed through the variable $\mathbf{x}$, i.e.,

$x_{1} \rightarrow \alpha x_{1}, x_{2} \rightarrow x_{2}, x_{3} \rightarrow \beta x_{3}-\gamma$

Using the notation defined in [3], set

$d=-2 a b+a b c+b c^{2}, e=\sqrt{b^{2} c^{2}\left(-4 a+a^{2}+2 a c+c^{2}\right)}, f=a b c+b c^{2}$,

$g=\sqrt{(d+e) / a}, h=\sqrt{(d-e) / a}$.

If $d<e$, system (27) has three equilibria, and if $d>e$, system (1) has five equilibria:

$S_{1}=[0,0,-\gamma]^{\mathrm{T}}, S_{2}=\left[\bar{x}_{1}, \bar{y}_{1}, \bar{z}_{1}\right]^{\mathrm{T}}, S_{3}=\left[-\bar{x}_{1},-\bar{y}_{1}, \bar{z}_{1}\right]^{\mathrm{T}}, S_{4}=\left[\bar{x}_{2}, \bar{y}_{2}, \bar{z}_{2}\right]^{\mathrm{T}}, S_{5}=\left[-\bar{x}_{2},-\bar{y}_{2}, \bar{z}_{2}\right]^{\mathrm{T}}$,

where

$$
\begin{aligned}
& \bar{x}_{1}=\sqrt{2} / 2 \alpha g, \bar{x}_{2}=\sqrt{2} / 2 \alpha h, \bar{y}_{1}=\sqrt{2} b c g \frac{a}{f+e}, \bar{y}_{2}=\sqrt{2} b c h \frac{a}{f-e}, \\
& \bar{z}_{1}=\frac{\beta(d+e) c}{f+e}-\gamma, \bar{z}_{2}=\frac{\beta(d-e) c}{f-e}-\gamma .
\end{aligned}
$$

\section{Energy cycling}

Note that the first term of the Qi chaotic system (27) is the torque transferred from the Hamiltonian energy; however, the dissipative and external torques are not related to forms of energy. Can we find corresponding energy terms related to the two types of torque? Morrison [22] extended the Lie-Poisson bracket of the Hamiltonian dynamics to include dissipation by a introducing a Lyapunov function $L$, i.e.,

$\dot{\mathbf{x}}=\{\mathbf{x}, H\}+\langle\mathbf{x},-L\rangle$,

with $\langle F, L\rangle=-g_{i j} \frac{\partial F}{\partial x_{i}} \frac{\partial L}{\partial x_{j}}$ (here the Einstein summation convention is used), $L$ is the Lyapunov function consisting of the dissipative power, and $g_{i j}$ is the Cartan-Killing metric: $g_{i j}=\frac{1}{2} \varepsilon_{i m}^{n} \varepsilon_{k n}^{m}$. Pelino, et al. [8] made use of the idea of an energy exchange between the Hamiltonian energy and dissipative energy, and further replaced $-L$ with $-L+G$, where $G$ is the supplied power 
transferred by the external force.

Following ideas extending the algebraic formalism of Hamiltonian dynamics to include dissipation and external force, we rewrite Eq. (27) as

$$
\dot{\mathbf{x}}=\{\mathbf{x}, H\}+\langle\mathbf{x},-L\rangle+\langle\mathbf{x}, G\rangle
$$

where $L=\frac{1}{2} \Lambda_{i} x_{i}^{2}, \quad G=f_{i} x_{i}, \quad i=1,2,3$,

$$
\langle\mathbf{x}, P\rangle=\frac{\partial P}{\partial \mathbf{x}}
$$

with $P$ representing either $L$ or $G$. Note that $\{\mathbf{x}, H\}=\mathbf{x} \times \frac{\partial H}{\partial \mathbf{x}}$, but $\langle\mathbf{x}, P\rangle=\frac{\partial P}{\partial \mathbf{x}}$. Therefore, $P$ must not represent the energy as $H$, but instead represents the power or rate of change in energy; that is why we define $2 L$ as the dissipative power and $G$ as the supplied power.

Eq. (33) can be interpreted as the total energy of the chaotic system containing the four different forms of energy: kinetic, potential, dissipative, and supplied, which correspond to the four different forms of torque: inertial, internal, dissipative, and external. For instance, in atmospheric circulation processes, an air particle contains kinetic energy, potential energy deriving from Earth's gravitational field, heat loss and radiant absorption. All the torque acting on the fluid particle contribute to $\dot{\mathbf{x}}$, the angular acceleration per unit of principle of inertia.

From Eqs. (3) and (34), we have

$$
\frac{\partial K}{\partial \mathbf{x}}\{\mathbf{x}, H\}=\{K, H\}, \frac{\partial K}{\partial \mathbf{x}}\langle\mathbf{x},-L\rangle=\langle K,-L\rangle
$$

Then we have

$$
\begin{aligned}
\dot{K} & =\frac{\partial K}{\partial \mathbf{x}} \dot{\mathbf{x}} \\
& =\frac{\partial K}{\partial \mathbf{x}}(\{\mathbf{x}, H\}+\langle\mathbf{x},-L\rangle+\langle\mathbf{x}, G\rangle) \\
& =\{K, H\}+\langle K,-L\rangle+\langle K, G\rangle \\
& =\{K, U\}+\langle K,-L\rangle+\langle K, G\rangle .
\end{aligned}
$$

Therefore, the rate of change in kinetic energy relates to its instantaneous exchange with the potential, dissipative, and supplied energy. Likewise, we have 


$$
\begin{aligned}
\dot{U} & =\{U, H\}+\langle U,-L\rangle+\langle U, G\rangle \\
& =\{U, K\}+\langle U,-L\rangle+\langle U, G\rangle,
\end{aligned}
$$

which implies that the rate of change in potential energy is equivalent to its instantaneous exchange with the kinetic, dissipative, and supplied energy. Also,

$\dot{H}=\langle H,-L\rangle+\langle H, G\rangle$

that is, the rate of change Hamiltonian is equivalent to its instant exchange with dissipative and supplied energy.

The Casimir function, like enstrophy or the potential vorticity in a fluid dynamical context, is very useful in analyzing stability conditions and the global description of a dynamical system [21]. The Casimir function $C$ is defined by the kernel of the bracket (3), i.e., $\{C, G\}=0, \forall G \in C^{\infty}\left(\mathbf{g}^{*}\right)$. Therefore it represents a constant of the motion of the Hamiltonian system, $\dot{C}=\{C, H\}=0$; that is, the Casimir function commutes under the Lie-Poisson bracket with every function [21]. It defines a foliation of the phase space $[8,19]$. The Casimir function for the Qi chaotic system is

$$
C=\frac{1}{2} \delta_{i j} x_{i} x_{j}
$$

From Eq. (3), $C$ satisfies the Casimir condition $\{C, G\}=0, \forall G \in C^{\infty}\left(\mathbf{g}^{*}\right)$. In addition, $C>0$ for $\forall x \neq 0$. From Eq. (33), we have

$$
\begin{aligned}
\dot{C} & =\{C, H\}+\langle C,-L\rangle+\langle C, G\rangle \\
& =\langle C,-L\rangle+\langle C, G\rangle \\
& =-2 L+G \\
& =-\left(a x_{1}^{2}+x_{2}^{2}+b\left(x_{3}+\gamma / 2\right)^{2}\right)+b \gamma^{2} / 4 .
\end{aligned}
$$

Remark 3: The Casimir function represents the existing internal energy of the system. The rate of change of the Casimir function is the exchange power between the supplied energy and the dissipative energy of the system. 2) The Casimir energy is invariant if the system neither loses energy nor absorbs energy from outside of the system.

To study the extremes of the Casimir function for the Qi chaotic system (27) or (33), let $\dot{C}=-\left(a x_{1}^{2}+x_{2}^{2}+b\left(x_{3}+\gamma / 2\right)^{2}\right)+b \gamma^{2} / 4=0$

Set $\Xi_{0}$ as a triaxle ellipsoid 
$\Xi_{0}=\left\{\mathbf{x} \mid a x_{1}^{2}+x_{2}^{2}+b\left(x_{3}+\gamma / 2\right)^{2}=b \gamma^{2} / 4\right\}$.

Regarding how the exchange power influences the dynamics of the Qi chaotic system, we have the following theorem:

Theorem 1:

(1) The extremes of the Casimir function is a triaxle ellipsoid $\Xi_{0}$; the extremes of system (27) and the origin belong to $\Xi_{0}$; the transpose of variable $\mathbf{x}$ is orthogonal to the vector field $\mathbf{u}=\dot{\mathbf{x}}$ on the surface of the ellipsoid $\Xi_{0}$.

(2) If $\dot{C}>0$, i.e., the supplied power is greater than the dissipative power, the orbits of the system stay inside the extremal ellipsoid, and the system stores internal energy. On the contrary, if $\dot{C}<0$, i.e., the supplied power is less than the dissipative power, the orbits of the system run outside the extremal ellipsoid, and the system releases internal energy. If $\dot{C}=0$, i.e., the supplied power counteracts the dissipative power, the system orbits intersect the extremal ellipsoid in the set $\Psi \cap \Xi_{\mathrm{o}}=\{\min (C), \max (C)\}$.

(3) The chaotic attractor is bounded, and the boundary sphere is

$\Xi=\left\{\mathbf{x} \mid x_{1}^{2}+x_{2}^{2}+(x-\gamma / 2)^{2}=b^{2} \gamma^{2} / 8(b-1)\right\}$

See proof in Appendix.

Finding the bound of a chaotic attractor is difficult [9] as the positive definite matrix in the Lyapunov stability equation is hard to obtain. In addition, the bound in [9] was found numerically instead of analytically as optimization software, such as LMI control toolbox, is needed in the solution process.

Remark 4: In proofing Theorem 1, the Casimir function property, i.e., $\{C, H\}=0$, has been used. This assumes an important role in finding the boundary sphere and rate of change of the internal energy.

Remark 5: The method used in analyzing the mechanics and energy cycling of the Qi chaotic attractor can be generalized to other numerical chaotic systems and physical chaotic systems with low dimension. However, the quadratic terms and linear terms of the selected system must be 
skew-symmetric; that is, the Lie-Poisson bracket must be satisfied.

\section{Analysis and illustration of energy cycle}

Because each type of torque is a coupled linear or nonlinear vector with three components acting on a particle or rigid body, the analysis of torque is quite difficult. However, energy is a scalar quantity that is easier to grasp. Conversely, because each type of torque has a corresponding energy, a study of energy can reflect torque characteristics. From here on, we study and illustrate the influence of the Casimir function in determining the system's chaotic behavior and the system's entire energy cycling.

Setting $a=35, b=\frac{8}{3}, c=80, \alpha=0.6, \quad$ we then obtain $\beta=\sqrt{1-\alpha^{2}}=0.8, \gamma=115.75, e_{1}=0.75$, $e_{2}=-2.083, e_{3}=1.333, c_{1}=107.8125$. We set $\mathbf{x}_{0}=\left[\begin{array}{lll}10 & -10 & 4\end{array}\right]$, and sampling time $T=0.001$. To understand the mechanism that produces chaos and energy cycling of system (33), we investigate the system's dynamics from simplicity to complexity.

\subsection{System under pure kinetic energy}

The system is described by

$\dot{\mathbf{x}}=\{\mathbf{x}, K\}$,

which only contains kinetic energy $K$ satisfying

$\dot{K}=\{K, K\}=0$.

Therefore the kinetic energy remains constant; i.e., the system is conservative and its value depends on the initial conditions of the orbit. From Eq. (44), the rate of change of the Casimir function vanishes

$\dot{C}=\{C, K\}=0$,

Because the energy described by the Casimir function is not exchanged with dissipative energy or supplied energy, the orbit remains periodic.

\subsection{System under inertial and internal torque}

The system is now described by 
$\dot{\mathbf{x}}=\{\mathbf{x}, K\}+\{\mathbf{x}, U\}$

There is conversion between kinetic energy and potential energy. From (36), the net rate of conversion of potential energy into kinetic energy is of the form

$\dot{K}=\{K, U\}=c_{1}\left(\Pi_{1}-\Pi_{2}\right) x_{1} x_{2}=-c_{1} e_{3} x_{1} x_{2}$.

Because $\dot{U}=-\{K, U\}$, the system is also conservative, i.e., kinetic energy lost is instantly gained in potential energy. From (46), the rate of change of the Casimir function vanishes

$\dot{C}=\{C, H\}=0$.

If the orbit is periodic, the system is also conservative. The question is how does this energy conversion work?.

Remark 6: 1) From Eq. (47), if in its orbit the system is located in quadrants I and III, $x_{1} x_{2}>0$, and there is a net conversion of kinetic energy into potential energy, i.e., $K \rightarrow U$ for $\dot{K}<0$; the opposite happens in quadrants II and IV where $x_{1} x_{2}<0$ corresponding to $U \rightarrow K$ for $\dot{K}>0$. Therefore, the plane $x_{1}=0$ or $x_{2}=0$ is the bound for this net conversion, in which the kinetic energy reaches its maximum or minimum for $\dot{K}=0$.

2) The net conversion rate of energy is proportional to the coefficient of potential energy, i.e., $c_{1}$, and $\Pi_{1}-\Pi_{2}=e_{3}$, and the product of angular momenta $x_{1}$ and $x_{2}$. It does not depend on $x_{3}$.

Fig. 1(a) clearly indicates how conversion works; here variables $x_{1}$ (blue one) and $x_{2}$ (red one) are in the top row, when either $x_{1}$ or $x_{2}$ crosses the zero line, i.e., the sign of $x_{1} x_{2}$ changes as indicated in the second row. When $\operatorname{sign}\left(x_{1} x_{2}\right)=-1$, corresponding to $\dot{K}>0$, i.e., kinetic energy increases as indicated in the third row (blue/red curve represents kinetic/potential energy), and vice versa. Fig. 1(b) shows that the periodic orbit running clockwise. When the system enters Quadrants I or III, the orbit changes color from red to blue, corresponding to an energy decrease, and vice versa in Quadrants II or IV. In the process, the conversion is fulfilled between kinetic energy and potential energy. On the vertical axis, $x_{1}=0$, the kinetic energy reaches a maximum, and on the 
horizontal axis, $x_{2}=0$, the kinetic energy drops to a minimum.
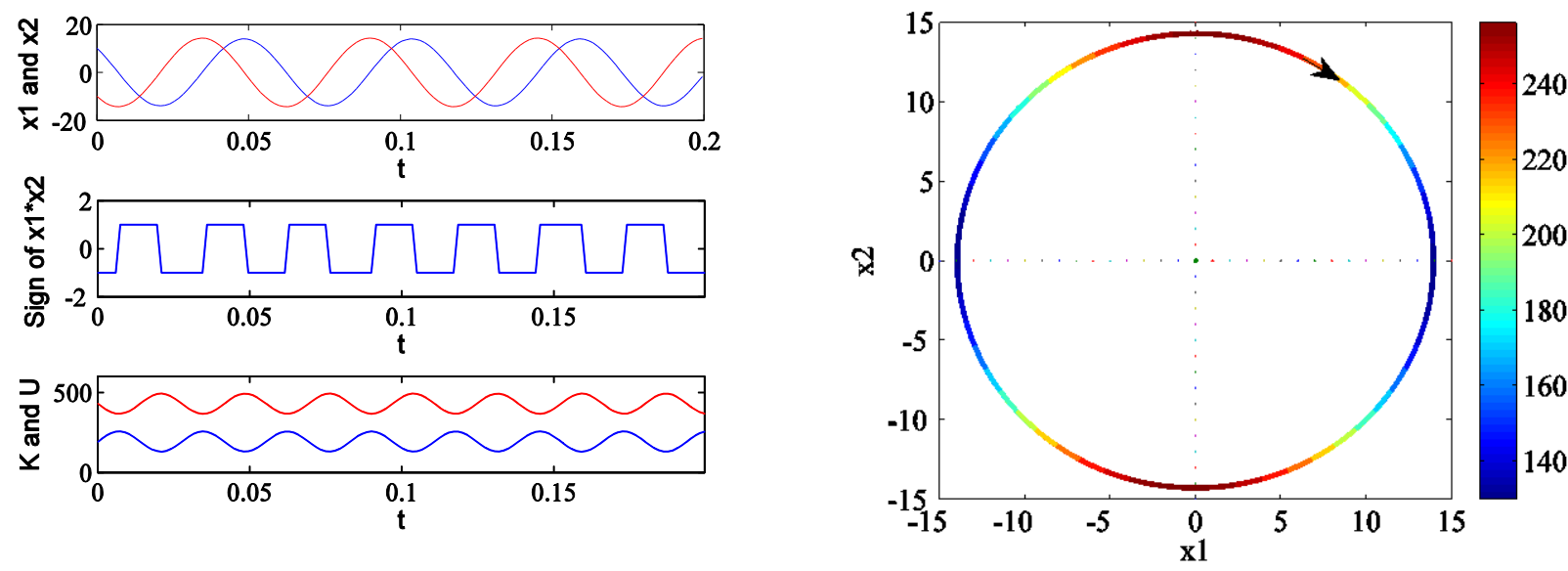

(a) Regulating the conversion between kinetic

(b) Kinetic energy around a periodic orbit. energy and potential energy.

Fig. 1. System is subject to inertial and internal torque.

In bifurcation analysis, a periodic orbit is often observed and plotted as the parameter is adjusted to a suitable value in the literature $[2,4,10]$. However, an analysis of the change and conversion in physical energy is missing because of the analytical methods applied. The energy-based method offers a tool to uncover energy running mechanisms that govern orbital behavior.

\subsection{System under inertial, internal, and dissipative torques}

Suppose a system is described by

$\dot{\mathbf{x}}=\{\mathbf{x}, H\}+\langle\mathbf{x},-L\rangle$.

Because only systems containing dissipation have fractal dimensions, dissipation is a necessary condition in producing a chaotic attractor. However, this is not sufficient condition to produce chaos. The dissipativity of the volume of system (49) is found to be

$\operatorname{Div}(\mathrm{V})=\frac{\partial \dot{x}_{1}}{\partial x_{1}}+\frac{\partial \dot{x}_{2}}{\partial x_{2}}+\frac{\partial \dot{x}_{3}}{\partial x_{3}}=-a-1-b$

where $V$ is the volume in the phase space of the system, with solution $V=V_{0} \exp (-(a+1+b) t)$. Therefore, system (49) is dissipative, i.e., the phase space volume shrinks exponentially to zero, provided $-(a+1+b)<0$. With the given parameter values, $-(a+1+b)=-38.667<0$, the system is dissipative. However, by calculation, the Lyapunov dimension $L_{d}=0$, and therefore the system 
has no fractal dimension and so does not produce chaos. Actually, the system produces a sink at the origin with Lyapunov exponents $L_{1}=-2.72, L_{2}=-17.95$, and $L_{3}=-17.99$. Using the Casimir function, we prove that the system produces a sink. The rate of change of the Casimir function is

$$
\dot{C}=\langle\mathbf{x},-L\rangle=-\left(a x_{1}^{2}+x_{2}^{2}+b x_{3}^{2}\right)<0 .
$$

Hence system (49) is globally asymptotically stable (Fig. 2) with a spiraling orbit converging onto the origin; the color of the curve represents the Hamiltonian energy (see color bar). The energy level of the system attractor diminishes from an initial high value to its final value (zero equilibrium). This reflects on the angular momentum (or moving speed) in the initial transient period where it is much larger than that during the steady state period close to the origin, and in particular very close to the origin in distance with slower speed.

Furthermore, we analyze the conversion between Hamiltonian energy and dissipative energy. From Eq. (25), we have

$$
H=K+U=\frac{1}{2}\left(x_{1}^{2}+\left(1+e_{3}\right) x_{2}^{2}+\left(1-e_{2}\right) x_{3}^{2}\right)+c_{1} x_{3} .
$$

As $\mathbf{x}$ converges to zero, the Hamiltonian energy decays to zero. From Eq. (51), the original Casimir energy is dissipated because the system contains dissipative terms, either friction or viscous forces, making the system finally losing its energy and stopping at the origin. We discuss in the next subsection what rate of change in the Casimir function could lead to chaos.

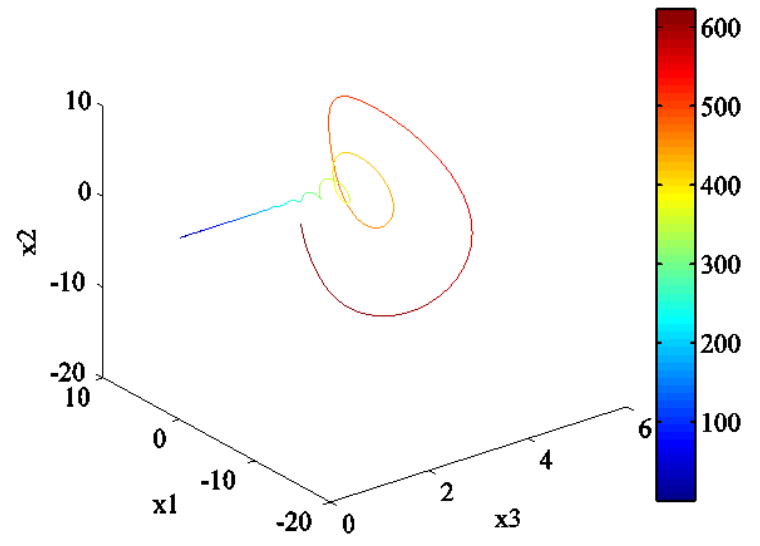

Fig. 2. Sink with Hamiltonian energy loss.

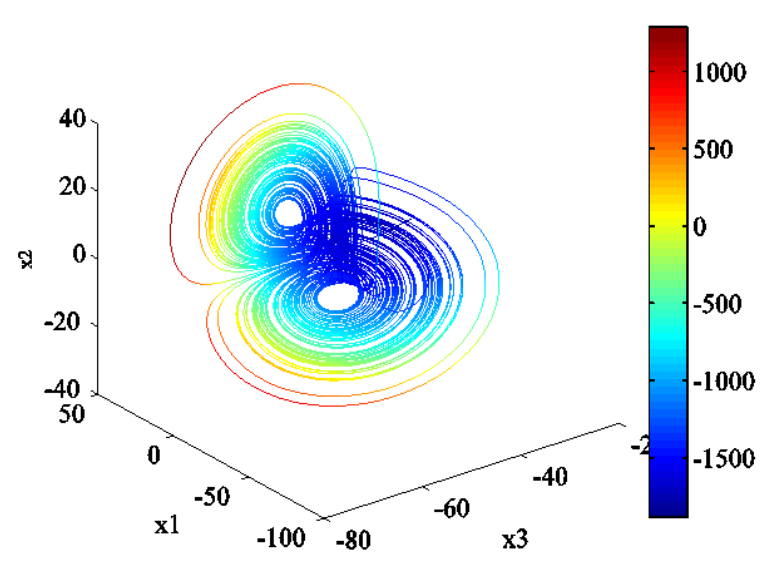

Fig. 3. 3D view of the Qi chaotic system.

\subsection{System under full torque}


Consider the following system

$\dot{\mathbf{x}}=\{\mathbf{x}, H\}+\langle\mathbf{x},-L\rangle+\langle\mathbf{x}, G\rangle$.

which differs from Eq. (49) by the addition of external torque $G=-b \gamma x_{3}$. We investigate how this term influences the rate of change of the Casimir function.

$\dot{C}=-2 L+G=-\left(a x_{1}^{2}+x_{2}^{2}+b\left(x_{3}+\gamma / 2\right)^{2}\right)+b \gamma^{2} / 4$.

From Eq. (53), there is an exchange of power between supplied energy and dissipative energy.

As a system without quadratic terms (inertial torque) is not nonlinear, no chaos is generated, and without dissipation a system cannot produce a fractal chaotic attractor. These two features are necessary to produce chaos; however, the two features cannot guarantee that a system generates chaos, as discussed in Section 4.3. To form a chaotic system is very complicated; for some systems, the internal torque and external torque are also important.

Remark 7: The rate of change of the Casimir function indicates the orbital mode of the system. If $\dot{C}<0$ holds for the system at all times, then the Casimir function of the system decays, and the orbit converges to a sink; a constant Casimir function, i.e., $\dot{C}=0$, signifies a conservative system; if $\dot{C}>0$ holds for the system at all times, the internal energy grows, and the orbit diverges; and if exchange power oscillates with bound, the system may produce chaos.

The chaotic attractor of the Qi system is illustrative (Fig. 3). The Lyapunov exponents are $L_{1}=4.1219, L_{1}=0, L_{1}=-42$, and the Lyapunov dimension is $L_{d}=2.0963<3$. Hence the system is chaotic and fractal. The Hamiltonian energy of the system attractor increases from the interior to the periphery, indicating that the particle angular momentum (or moving speed) along the periphery is much larger than that close to the center of the attractor. In addition, note that the energy in the lower left is high (red) whereas that in the upper right is low (blue). A reason for this is disclosed later.

Both kinetic energy and potential energy oscillate with bounds and are exchanged (Fig. 4) When the kinetic energy peaks, the potential energy is a minimum. The rate of change of the Casimir function oscillates around the zero line (Fig. 5) indicating the value of Casimir increases or decreases. 


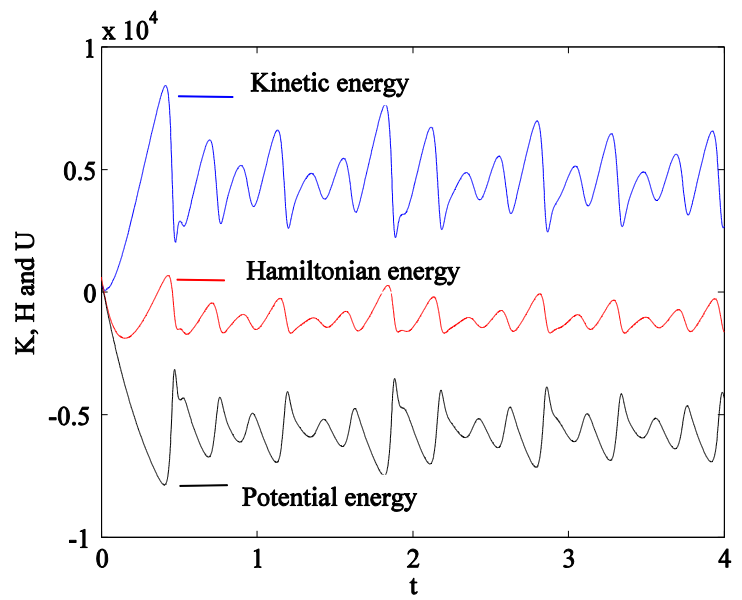

Fig. 4. Kinetic, Hamiltonian, and potential energy
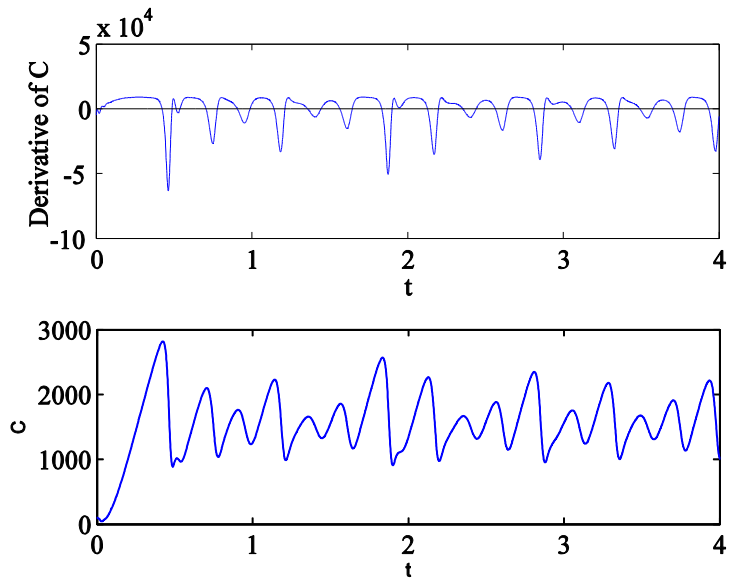

Fig. 5. Casimir function and its derivative. of the Qi chaotic system.

We now illustrate Theorem 1 in three parts corresponding to the three parts of the theorem.

1) From Eq. (29), $d-e=-56.46$, and hence the system has three equilibria $S_{1}, S_{2}$, and $S_{3}$ as given in Eq. (30). The Qi chaotic attractor intersects the ellipsoid generated from the extremal points of the Casimir function (Fig. 6) where the three equilibria are seen on the ellipsoid: $S_{1}$ (blue star) is located on the bottom end of the $x_{3}$-axis of $\Xi_{0}$, and $S_{2}$ and $S_{3}$ (black '+') are at the centers of the wings of the chaotic attractor intersecting the waist of the ellipsoid. The origin is at the top end of the $x_{3}$-axis of $\Xi_{0}$. Note that to display the equilibria and the symmetric property, Fig. 6 has been rotated. Because system (27) has the symmetry, $x_{i} \rightarrow-x_{i}, i=1,2$, the chaotic attractor intersects ellipsoid $\Xi_{0}$ symmetrically. Refs. [8] and [17] investigated the invariant ellipsoid and the energy cycle of the Lorenz chaotic attractor using the Casimir function.
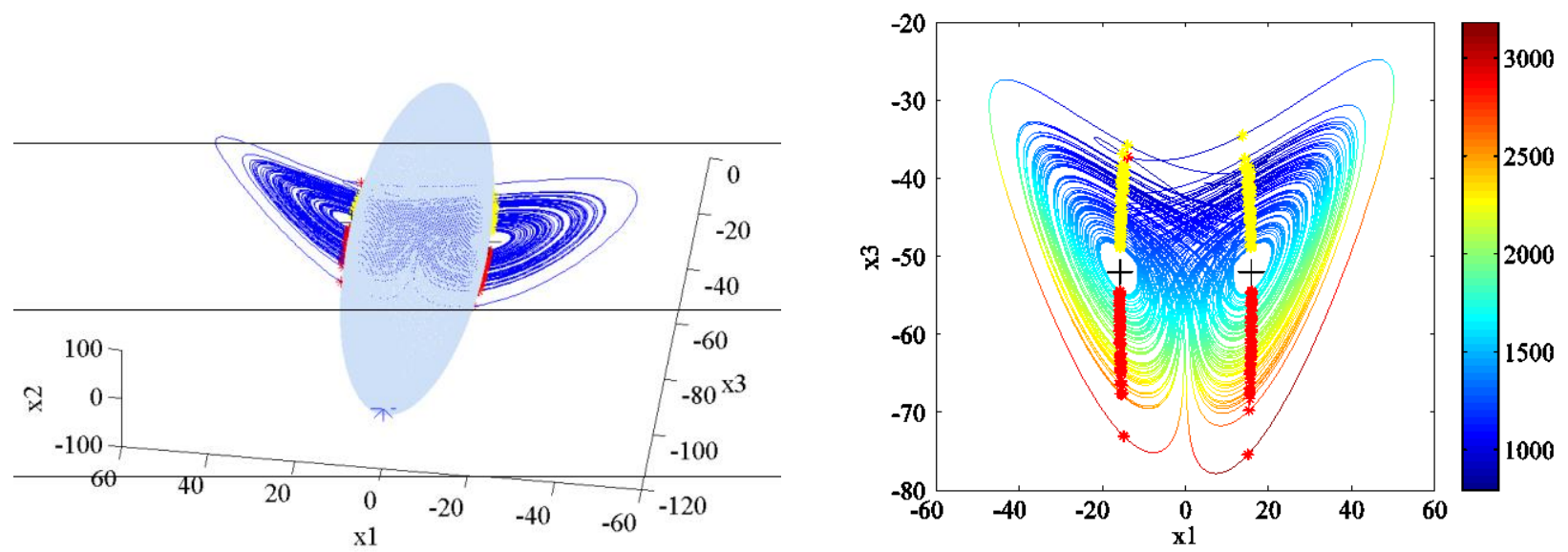

attractor with three equilibria.

2) Although the system is governed by Eq. (27) or (53), the core energy of the Casimir function, like enstrophy or potential vorticity, has an impact on the system to some extent. The ellipsoid represents the contour surface $\dot{C}=0$ where the exchange power is zero. When the trajectories of the system exit from inside the ellipsoid (red star in Fig. 6), they intersect points at which the derivative of the Casimir function decreases in traversing the zero line (first curve of Fig. 5); hence the Casimir function peaks (second curve in Fig. 5). Thereafter, the dissipative power is smaller than the supplied power, i.e., $\dot{C}<0$. When the energy drops to its minimum, the trajectories reenter the ellipsoid (yellow star in Fig. 6). The frequency of the derivative of the Casimir function across the zero line equals the number of intersections the chaotic orbit has with the ellipsoid. To clearly display the oscillation of Casimir functions, we remove the ellipsoid but retain the sign for each traversal (red and yellow stars in Fig. 7) in an $x_{1}-x_{3}$ projection; the color bar gives Casimir energy in the range [792.5,3181]. Generally, there are two regions colored red and blue. The energy of the orbit around red stars (maximum sign) are higher than those around yellow stars (minimum sign). In each cycle, we find that the orbit runs from right wing starting from maximum Casimir energy (red star), and losses energy until reaching a minimum (yellow star). The orbit then gradually moves to the left wing with increasing Casimir energy reaching its maximum (red star), then decreasing to its minimum (yellow star). Finally, the orbit returns to the right wing. The orbit cycles repeatedly as the Casimir energy oscillates. The Casimir ellipsoid is the ideal Poincaré map.

3) The chaotic attractor (red orbit in Fig. 8) is bounded and from Eq. (43) is contained in the sphere $\Xi$ of radius $r=b \gamma /(2 \sqrt{2(b-1)})=84.5318$.

Chaotic systems come with various established properties: sensitivity to initial conditions, fractal dimension, instability, and boundary solution. For a non-chaotic system, positive Lyapunov exponents imply the system is unstable and the solution grows without bound. However, a chaotic attractor is bounded, so the trajectory repeatedly grows in fractal dimension with folding and stretching. Therefore the boundary solution property is important in chaotic dynamics. Clearly, 
Fig. 8 presents not the least bounds. Can we find the least upper bound, i.e., the supremum?

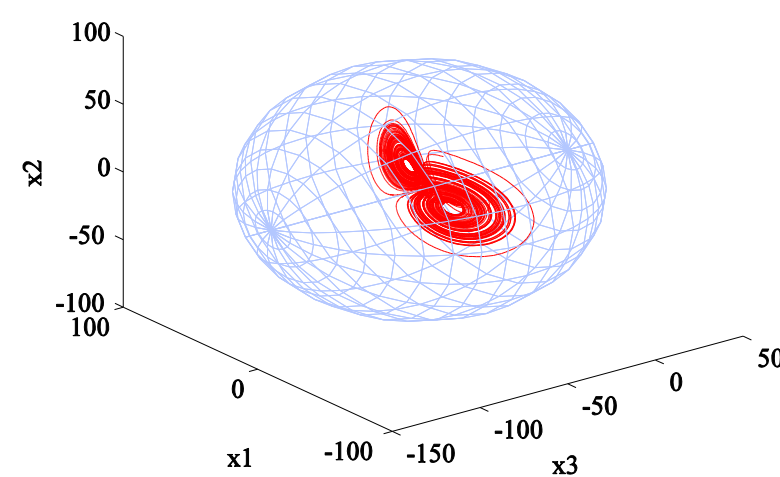

Fig. 8. Boundary sphere of chaotic attractor.

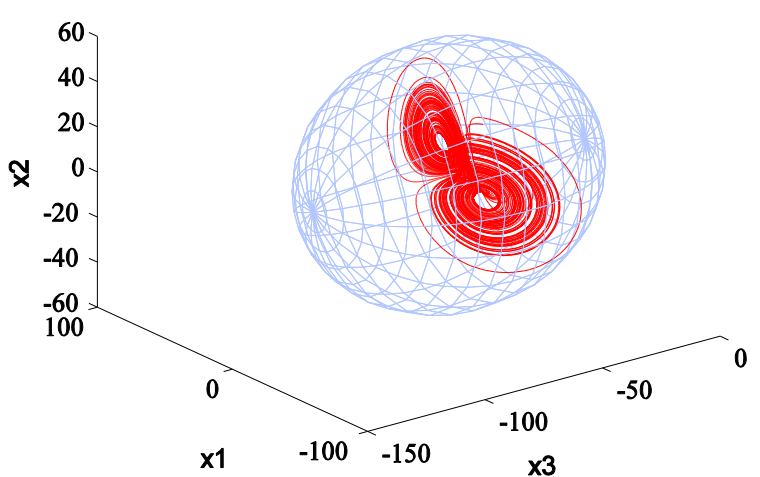

Remark 8: The supremum of the chaotic system (27) is the sphere,

$\Xi_{s}=\left\{\mathbf{x} \mid x_{1}^{2}+x_{2}^{2}+\left(x_{3}-\gamma / 2\right)^{2}=\gamma^{2} / 4\right\}$.

Currently, we have no rigorous proof. Therefore, we leave this as an open problem. Here we shall content ourselves just to explain it. From Theorem 1, the ellipsoid is $x_{3}$-axial symmetric with the origin on the top end of the $x_{3}$-axis of $\Xi_{0}$; its equilibrium $S_{1}=[0,0,-\gamma]^{\mathrm{T}}$ is on the bottom end of the $x_{3}$-axis of $\Xi_{0}$, which is the set of extremals including all minimum and maximum of $C$. For any maximum $C_{m}$, the Casimir function is given by the sphere $\frac{1}{2}\left(x_{1}^{2}+x_{2}^{2}+x_{3}^{2}\right)=C_{m}$ with center at the origin and radius $\sqrt{2 C_{m}}$. When the largest maximum $C_{s}$ is found, $x_{1}^{2}+x_{2}^{2}+x_{3}^{2}=2 C_{s}$ is the least bound sphere. Clearly, $C_{s}$ is on the ellipsoid $\Xi_{0}$. Therefore we need to find the point on $\Xi_{0}$ furthest from the origin. For the given parameters of the system, the point is the equilibrium $S_{1}$ at the bottom end of the $x_{3}$-axis of $\Xi_{0}$, and $\left\|s_{1}\right\|=\gamma$, that is $2 C_{s}=\gamma^{2}$. Therefore the bound is a sphere with center at origin and radius $\gamma$, i.e., $x_{1}^{2}+x_{2}^{2}+x_{3}^{2}=\gamma^{2}$. Because both the chaotic attractor and ellipsoid $\Xi_{0}$ are centered at $[0,0,-\gamma / 2]^{\mathrm{T}}$, to get the supremum of chaotic attractor, we shift the spherical center from origin to $[0,0,-\gamma / 2]^{\mathrm{T}}$. As a consequence, the sphere radius can be reduced to $\gamma / 2$. Therefore, the final supremum of chaotic attractor is $\Xi_{s}$, i.e., as Eq. (55). If the 
initial conditions of the system are $\mathbf{x}_{0} \in \Xi_{s}, \mathbf{x}(t) \in \Xi$ for all $t>0$; if $\mathbf{x}_{0} \notin \Xi_{s}, \lim _{t \rightarrow \infty} \mathbf{x}(t) \in \Xi_{s}$. The supremum sphere just encloses the attractor (Fig. 9) whereas the sphere bounding the attractor in Fig. 8 is larger.

From Fig. 4, we know that the Hamiltonian energy is not conserved when the system produces chaos. We discuss next the influence of the Hamiltonian energy on the chaotic system. From Eq. (38), we find

$$
\begin{aligned}
\dot{H}= & \langle H,-L\rangle+\langle H, G\rangle \\
= & -a x_{1}^{2}-\left(1+e_{3}\right) x_{2}^{2}-b\left(1-e_{2}\right) x_{3}^{2} \\
& -\left(b \gamma\left(1-e_{2}\right)+b c_{1}\right) x_{3}-b c_{1} \gamma \\
= & -a x_{1}^{2}-\left(1+e_{3}\right) x_{2}^{2}-b\left(1-e_{2}\right)\left(x_{3}+\frac{b \gamma\left(1-e_{2}\right)+b c_{1}}{2 b\left(1-e_{2}\right)}\right)^{2}+\frac{\left(b \gamma\left(1-e_{2}\right)+b c_{1}\right)^{2}}{4 b\left(1-e_{2}\right)}-b c_{1} \gamma .
\end{aligned}
$$

For nonzero values, energy conservation is lost and begins to oscillate with the exchange between dissipative energy and supplied energy. Setting $\dot{H}=0$ determines the Hamiltonian conservative ellipsoid $\Xi_{H}$ (Fig. 10). The orbit of the Qi chaotic attractor enters the ellipsoid (twice for the right and left wings) when the Hamiltonian function descends to its minimum, and thereafter the orbit moves inside the ellipsoid; symmetrically, it exits (twice for the left and right wings) when the Hamiltonian function ascends to its maximum, and thereafter the orbit moves outside the ellipsoid; the cycle then repeats. The chaotic attractor is bounded, and the boundary sphere is $\Xi_{H}=\left\{\mathbf{x} \mid a x_{1}^{2}+\left(1+e_{3}\right) x_{2}^{2}+b\left(1-e_{2}\right)\left(x_{3}+\phi\right)^{2}=r_{H}^{2}\right\}$

where $\phi=\frac{b \gamma\left(1-e_{2}\right)+b c_{1}}{2 b\left(1-e_{2}\right)}$, and $r_{H}^{2}=\frac{\left(b \gamma\left(1-e_{2}\right)+b c_{1}\right)^{2}}{4 b\left(1-e_{2}\right)}-b c_{1} \gamma$.

Similar to the Casimir function, the Hamiltonian energy begins to oscillate; over one cycle, its energy peaks when the orbit exits from the ellipsoid on the right wing (red star) and then it gradually decreases, then reenters the conservative ellipsoid on the right wing (yellow star) when the Hamiltonian energy tends to its minimum. The change in energy governs the swing in orbit from right to left wing. From Fig. 3, we noted earlier the color difference associated with the Hamiltonian energy; this is caused by its oscillations being red for maximum energy and blue for minimum energy. Although the Hamiltonian energy oscillates, the change in energy is not periodic ( Fig. 11); higher energies govern the circular orbits farther out in the two wings, whereas lower energies 
dictate smaller circular orbits. This difference makes the chaotic system aperiodic and sensitive to initial conditions.

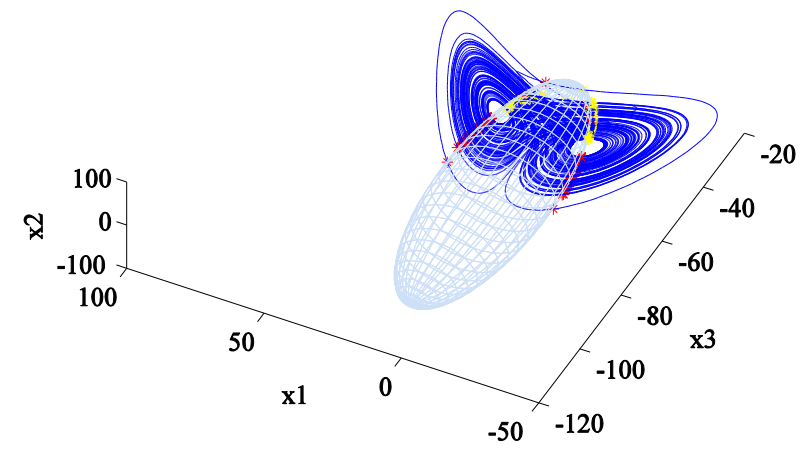

Fig. 10. Hamiltonian conservative ellipsoid.

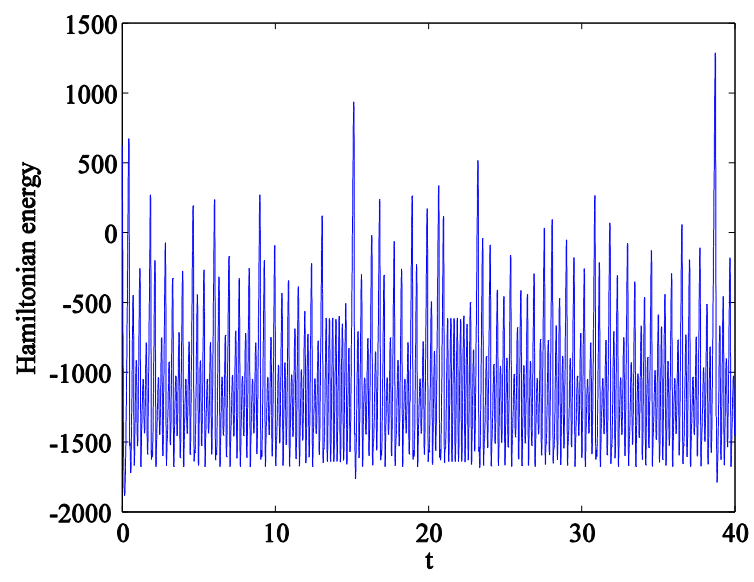

Fig. 11. Time series of Hamiltonian energy.

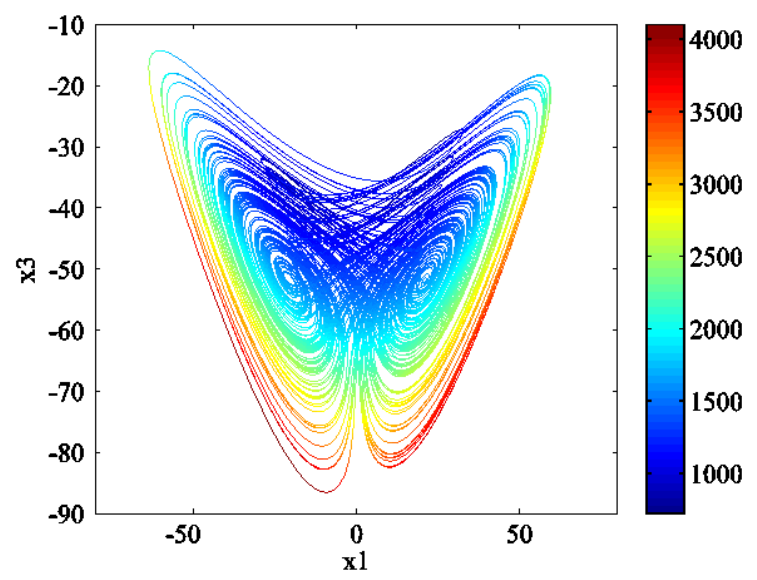

Fig. 12. Phase portrait with $b \gamma=1.4 b \gamma$.

We continue with the effect external torque has on the chaotic attractor. From Eq. (55), the radius of the supremum sphere is related to the magnitude of the external torque. In addition, from Eq. (42), the extremal ellipsoid intersecting with the attractor is also impacted by the external torque. When the factor associated with external torque is increased 1.4 times, i.e., $b \gamma=1.4 b \gamma$, the attractor changes as well (Fig. 12). First, the range for the Casimir function expands to [722.6, 4100] from [792.5, 3181], and the frequency associated with the derivative of the Casimir function crosses the zero line, i.e., the frequency of intersection increases from 351 to 501 in a period of 40 time units, therefore the oscillation between the two wings has increased. Second, the orbits are much denser 
than those in Fig. 7 because the orbits of the new chaotic attractor frequent the holes around equilibria, despite there being quite empty regions in the original attractor (Fig. 7); the magnitude of the torque has increased as well. Third, the Lyapunov property has changed from $L_{1}=4.1219$, $L_{d}=2.0963$ to $L_{1}=6.784, L_{d}=2.149$. Therefore, the degree of randomness is enhanced and the geometric space the orbit occupies has increased.

\section{Conclusion}

The Qi chaotic system was transformed into the Kolmogorov type system to investigate the mechanics of the system. The physical interpretation of the state variable was identified. Four forms of energy for the Qi system were uncovered: kinetic, potential, dissipative, and supplied. The exchange between dissipative power and supplied power was found using the Casimir function to determine the dynamic modes of the system. The general bound and least bound of the Qi chaotic system were given in terms of the Casimir function. A detailed analysis and illustrations for combinations of the four forms of energy further revealed insights and contributing factors concerning the dynamics of periodic orbits, sinks and chaos.

\section{References}

[1] Chen G, Ueta T. Yet another chaotic attractor. Int. J. Bifurc. Chaos 1999; 9:1465-1466.

[2] Lü J, Chen G. A new chaotic attractor coined. Int. J. Bifurc. Chaos 2002; 12:659-661.

[3] Qi G, G. Chen, S. Du, Z. Chen, Z. Yuan. Analysis of a new chaotic system. Physica A: Statistical Mechanics and its Applications 352 (2005) 295-308.

[4] Qi G, van Wyk M A, van Wyk B J, Chen G. On a new hyperchaotic system. Phy. Lett. A 2008; 372:124-136.

[5] Shen C, Yu S, Lü J, Chen G. Designing hyperchaotic systems with any desired number of positive Lyapunov exponents via a simple model. IEEE Trans. Circuits and Syst. I 2014; 61:2380-2389.

[6] Lorenz E. Deterministic nonperiodic flow. Journal of the Atmospheric Sciences 1963; 20:130-141.

[7] Rodríguez J L D, Thompson M. A balanced atmospheric model of Lorenz. Nonlinear Anal: RWA 2010; 11:3251-3271.

[8] Pelino V, Maimone F, A. Pasini Energy cycle for the Lorenz attractor. Chaos, Solitons \& Fractals 2014; 64: 67-77.

[9] Wang P, LI D, WU X, Lü J, Yu X. Ultimate bound estimation of a class of high dimensional quadratic autonomous dynamical systems. Int. J. Bifurc. Chaos 2011; 21: 2679-2694.

[10] Yang J, Zhao L. Bifurcation analysis and chaos control of the modified Chua's circuit system. Chaos, Solitons \& Fractals. 2015; 77:332-339.

[11] Qi G, Wang Z, Guo Y. Generation of an eight-wing chaotic attractor from Qi 3-D four-wing chaotic system. 
Int. J. Bifurc. Chaos 2012; 22:1250287-1-9.

[12] Qi G, Chen G, van Wyk M A, van Wyk B J, Zhang Y. A Four-wing chaotic attractor generated from a new 3-D quadratic chaotic system. Chaos Solitons \& Fractals 2008; 38:705-721.

[13] Das S, Pan I, Das S. Effect of random parameter switching on commensurate fractional order chaotic systems. Chaos, Solitons \& Fractals. 2016: 91:157-173.

[14] Bodale I, Oancea V A. Chaos control for Willamowski-Rössler model of chemical reactions. Chaos, Solitons \& Fractals 2015; 78:1-9.

[15] Arnold V. Kolmogorov Hydrodynamic attractors. Proceedings of Royal Society of London 1991; 434:19-22.

[16] Pasini A, Pelino V. A unified view of Kolmogorov and Lorenz systems. Phys. Lett. A. 2000; 275: 435-446.

[17] M. Gianfelice1, F. Maimone, V. Pelino, S. Vaienti. On the Recurrence and Robust Properties of Lorenz'63 Model. Commun. Math. Phys. 313, 745-779 (2012).

[18] Qi G, Liang X. Mechanical analysis of Qi four-wing chaotic system. Nonlinear Dynamics, 2016, 86(2): 1095-1106.

[19] Arnold V I, Khesin B A. Topological methods in hydrodynamics. Springer, Berlin, 1998.

[20] V Pelino, F Maimone. Energetics, skeletal dynamics, and long-term predictions on Kolmogorov-Lorenz systems. Physical Review E Statistical Nonlinear \& Soft Matter Physics, 2007, 76(2):70-80.

[21] Marsden J E, Ratiu T S. Introduction to Mechanics and Symmetry: A Basic Exposition of Classical Mechanical Systems. 2nd Edition, Springer, 2002. Chapter 1.

[22] Morrison P J. Thoughts on brackets and dissipation: old and new. Journal of Physics: Conference Series 2009; 169:1-12.

\section{Appendix}

\section{Proof of Theorem 1}

(1) Setting $\dot{C}=0$, then $\Xi_{0}$ is a triaxle ellipsoid formed by the fixed points of the Casimir function. The ellipsoid is obtained by solving $\dot{C}=\frac{\partial C}{\partial \mathbf{x}} \dot{\mathbf{x}}=0$. Because the system's equilibria are obtained by solving $\dot{\mathbf{x}}=0$, the three equilibria lie on $\Xi_{0}$. If $\mathbf{x}^{\mathrm{T}}=\frac{\partial C}{\partial \mathbf{x}}=0$, we have $\dot{C}=0$, so the origin is also on $\Xi_{0}$. The ellipsoid is $x_{3}$-axial symmetric, and its origin is at the top end of the $x_{3}$ -axis of $\Xi_{\mathrm{o}}$ with equilibrium $S_{1}=[0,0,-\gamma]^{\mathrm{T}}$ at the bottom end. Because $\dot{C}=\frac{\partial C}{\partial \mathbf{x}} \dot{\mathbf{x}}=\mathbf{x}^{\mathrm{T}} \dot{\mathbf{x}}=0$, the transpose of $\mathbf{x}$ is orthogonal to the vector field $\mathbf{u}=\dot{\mathbf{x}}$ on $\Xi_{0}$.

(2) If $\dot{C}>0$, from Eq. (40), we have $-2 L+G>0$, so the supplied power is greater than the dissipative power; hence internal energy is stored. Further, we have 
$a x_{1}^{2}+x_{2}^{2}+b\left(x_{3}+\gamma / 2\right)^{2}<b \gamma^{2} / 4$, the orbit of the system stays inside $\Xi_{0}$; Likewise, if $\dot{C}>0$, we have $-2 L+G<0$, so the supplied power is less than the dissipative power; hence the internal energy is released, and $a x_{1}^{2}+x_{2}^{2}+b\left(x_{3}+\gamma / 2\right)^{2}>b \gamma^{2} / 4$, the orbit of the system moves outside $\Xi_{0}$. If $\dot{C}=0$, i.e., the supplied power counteracts the dissipative power, the system's orbits transit the extremal ellipsoid in the set $\Psi \cap \Xi_{\mathrm{o}}=\{\min (C), \max (C)\}$, and the cycle repeats.

(3) The following inequality can be obtained

$\dot{C}=-a x_{1}^{2}-x_{2}^{2}-b x_{3}^{2}-b \gamma x_{3}$

$\leq-a x_{1}^{2}-x_{2}^{2}-b x_{3}^{2}+(b-1) x_{3}^{2}+b^{2} \gamma^{2} / 4(b-1)$

$\leq-\left(x_{1}^{2}+x_{2}^{2}+x_{3}^{2}\right)+b^{2} \gamma^{2} / 4(b-1)$.

$=-2 C+b^{2} \gamma^{2} / 4(b-1)$.

Here the following inequality is used.

$b \gamma x_{3}=-\sqrt{2(b-1)} x_{3} \frac{b \gamma}{\sqrt{2(b-1)}} \leq(b-1) x_{3}^{2}+\frac{b^{2} \gamma^{2}}{4(b-1)}$.

From (39), we have

$C \leq e^{-2 t}\left(C_{0}+\int_{0}^{t} e^{2 \tau} \frac{b^{2} \gamma^{2}}{4(b-1)} d \tau\right)$

When $t \rightarrow \infty$,

$C \leq \frac{b^{2} \gamma^{2}}{2(b-1)}+\varepsilon$,

where $\lim _{t \rightarrow \infty} \varepsilon=0$. Let $\Xi=\left\{\mathbf{x} \mid x_{1}^{2}+x_{2}^{2}+x_{3}^{2}=b^{2} \gamma^{2} /(b-1)\right\}$. For both the chaotic attractor and ellipsoid $\Xi_{0}$ centered at $[0,0,-\gamma / 2]^{\mathrm{T}}$, we shift the center of sphere $\Xi$ from the origin to $[0,0,-\gamma / 2]^{\mathrm{T}}$ to set the bound for the attractor. In consequence, the radius of the sphere is half the size, i.e., $b \gamma /(2 \sqrt{2(b-1)})$. Therefore, the final bounding sphere is $\Xi$, i.e., Eq. (43). When the initial state of the system $\mathbf{x}_{0} \in \Xi$, then $\mathbf{x}(t) \in \Xi$ for all $t>0$; when $\mathbf{x}_{0} \notin \Xi, \lim _{t \rightarrow \infty} \mathbf{x}(t) \in \Xi$. Therefore, the sphere $\Xi$ is the bound of the Qi chaotic attractor. The proof is complete. 